\title{
SARS-CoV-2 Slows Brain Rhythms with more Severe Effects in Younger Individuals
}

\section{Carl Saab ( $D$ CARL_SAAB@brown.edu )}

Cleveland Clinic https://orcid.org/0000-0003-4665-9946

\section{Helen Valsamis}

King's County and SUNY Health Sciences University

\section{Samah Baki}

King's County and SUNY Health Sciences University

Jason Leung

Cleveveland Clinic

\section{Samer Ghosn}

Cleveland Clinic

\section{Brittany Lapin}

Cleveland Clinic

\section{Geetha Chari}

King's county

Izad-Yar Rasheed

King's county

Jaehan Park

King's county

\section{Vineet Punia}

Cleveland Clinic

\section{Dileep Nair}

Cleveland Clinic Neurological Institute

Ann Marie Kaniecki

Cleveland Clinic

\section{Muhammad Edhi}

Cleveland Clinic

\section{Article}

\section{Keywords:}

Posted Date: January 12th, 2022 
DOI: https://doi.org/10.21203/rs.3.rs-1197196/v1

License: (a) (i) This work is licensed under a Creative Commons Attribution 4.0 International License. Read Full License 
Title: SARS-CoV-2 slows brain rhythms with more severe effects in younger individuals

Authors: Helen Valsamis',2, Samah Abdul Baki2,3, Jason Leung, Samer Ghosn³, Brittany Lapin ${ }^{4}$, Geetha Chari1,2, Izad-Yar Rasheed ${ }^{1}$, Jaehan Park ${ }^{1,2}$, Vineet Punia ${ }^{4}$, Dileep Nair ${ }^{4}$, Ann Marie Kaniecki ${ }^{4}$, Muhammad Edhi $^{4}$, Carl Y Saab4,5,6

1 King's County, Brooklyn, NY

2 SUNY Health Sciences University, Brooklyn, NY

3 BioSignal Group Corp, Acton, MA

4 Cleveland Clinic Foundation, Cleveland, $\mathrm{OH}$

5 Case Western Reserve University, Cleveland, $\mathrm{OH}$

6 Brown University, Providence, RI

\begin{abstract}
Coronavirus disease secondary to infection by SARS-CoV-2 (COVID19 or C19) causes respiratory illness, as well as severe neurological symptoms that have not been fully characterized. In a previous study, we developed a computational pipeline for the automated, rapid, high-throughput and objective analysis of brain encephalography (EEG) rhythms. In this retrospective study, we used this pipeline to define the quantitative EEG changes in patients with a PCR-positive diagnosis of $C 19(n=31)$ in the intensive care unit (ICU) of Cleveland Clinic, compared to a group of age-matched PCR-negative $(n=38)$ control patients in the same ICU setting. Qualitative assessment of EEG by two independent teams of electroencephalographers confirmed prior reports with regards to the high prevalence of diffuse encephalopathy in C19 patients, although the diagnosis of encephalopathy was inconsistent between teams. Quantitative analysis of EEG showed distinct slowing of brain rhythms in C19 patients compared to control (enhanced delta power and attenuated alphabeta power). Surprisingly, these C19-related changes in EEG power were more prominent in patients below age 70. Moreover, machine learning algorithms showed consistently higher accuracy in the binary classification of patients as C19 versus control using EEG power for subjects below age 70 compared to older ones, providing further evidence for the more severe impact of SARS-CoV-2 on brain rhythms in younger individuals irrespective of PCR diagnosis or symptomatology, and raising concerns over potential long-term effects of C19 on brain physiology in the adult population and the utility of EEG monitoring in C19 patients.
\end{abstract}




\section{Introduction}

Coronavirus disease (COVID19, abbreviated here as C19) is caused by infection with the SARS-CoV-2 virus. Most people infected with the virus will experience mild to moderate respiratory illness, and individuals with underlying medical conditions, especially older people, appear to be more vulnerable (World Health Organization, https://www.who.int/health-topics/coronavirus\#tab=tab_1). Irrespective of these risk factors, and for incompletely understood reasons, some will become seriously ill and manifest severe neurological symptoms requiring admission to an intensive care unit (ICU). Although evidence related to the presence of SARS-CoV-2 in the central nervous system (CNS) is sparse, and direct viral invasion of the CNS is difficult to estimate [1,2], the significant impact of the virus on the CNS and its contribution to neurological sequelae are uncontested, partly as a result of immune-mediated or autoimmune reactions leading to neuro-inflammation [3].

Neurological symptoms of C19 include loss of smell and seizures [4, 5], and more broadly defined symptoms such as brain 'fogginess', dizziness, extreme fatigue, and sleepiness [6,7]. In cases where electroencephalography (EEG) has been performed on C19 patients, predominant observations converge on generalized slowing and diffuse encephalopathy [8-19]. Invariably, however, these observations have been based on visual interpretation of the EEG, a method that is known to be qualitative and time consuming. Conventional EEG systems require significant training and expertise for accurate visual interpretation of EEG, in particular for the removal of artifacts which exacerbate inter-rater variability and sampling bias [20-22]. These concerns are more salient when dealing with C19 patients, especially in the ICU. Even though subjective interpretation of EEG led many studies to conclude that EEG patterns are significantly altered in C19 patients [8-19], primarily in frontal lobes [8, 12,19] and within distinct low frequency bands of delta [12] and alpha [15], detailed, quantitative and objective characterization of these patterns has only been achieved in two studies with low sample sizes [14, 23]. A few studies have even suggested that EEG is visually normal with scarce abnormalities [24] or indistinguishable from other pathological conditions [25]. Moreover, widely held assumptions about milder neurologic symptoms in the pediatric population have been challenged [26, 27], and the lack of adequate control groups in several EEG studies is a major concern [14], especially with regards to age which is a significant variable in EEG research $[28,29]$. Hence, there is an urgent unmet need for the standardization of quantitative EEG analysis for the discovery of objective, physiological markers that could help in the diagnosis, prognosis and monitoring of therapy in critically ill patients with C19 [14, 23, 24].

Rhythmic brain activity or 'oscillation' in discrete frequency bands, a fundamental characteristic of spontaneous activity in mammalian brain covering large scale neural networks, is a hallmark of mental states in health [30-32] and disease [33]. The study of spontaneous brain oscillations reveals subtle, emergent properties of dynamic and high-speed brain function related to mental states in real-time. Therefore, we postulated that the study of EEG oscillations in C19 patients with CNS symptoms could potentially lead to the discovery of novel markers of the disease. A comprehensive and detailed assessment of oscillations, including power amplitude in distinct frequency bands and temporal coupling between bands, cannot be achieved reliably by visual inspection alone. Therefore, our laboratory has developed a computational pipeline for the automated, rapid, high-throughput and objective analysis of EEG oscillations. This pipeline is based on a support vector machine (SVM) algorithm for the accurate detection of EEG artifacts, which has been validated in rodent, non-human primate and human subjects [34].

In this retrospective study including 69 subjects, we investigated the quantitative changes in EEG oscillations of patients with a PCR-positive diagnosis of C19 $(n=31)$ in the ICU at Cleveland Clinic, compared to a group of PCR-negative $(n=38)$ patients in the same ICU. Clinical reviews of EEG by visual inspection conducted by clinical fellowship trainees and board certified electroencephalographers at two independent sites (Cleveland Clinic, $\mathrm{OH}$ and King's County/SUNY, NY) confirmed prior reports with regards to the high prevalence of diffuse encephalopathy in C19 patients, albeit to the same degree observed in age-matched control patients. Moreover, diagnoses of encephalopathy at the individual subject level were not fully consistent across both sites. Quantitative EEG analysis, however, showed distinct slowing of EEG in C19 subjects compared to control subjects, which was more severe in subjects below age 70, such that power distribution in younger subjects in the low frequency bands $(<35 \mathrm{~Hz})$ was indistinguishable from that in subjects above age 70. These results were further corroborated using an unbiased approach based on machine learning, whereby the accuracy of several algorithms for the binary classification of patients as C19 versus control using EEG power features was consistently higher for patients below age 70, raising concern about the long-term effects of C19 in younger age population and the utility of EEG monitoring in C19 patients of all ages. 


\section{Results}

Visual interpretation of EEG by two independent teams of trained electroencephalographers at Cleveland Clinic and SUNY resulted in normal or encephalopathy diagnosis (Table 2). Overall, this qualitative diagnosis was inconsistent among both teams, and more so for the C19 group (14 inconsistent diagnoses out of 31 or $45 \%$ ) versus control (7 inconsistent diagnoses out of 38 or $22 \%$ ). When subjects were age-matched (Sup Fig 1), quantitative analysis of the mean EEG power showed distinct and significant differences in C19 compared to the control group (Fig 1), including an increase in delta $(0.5 \pm 0.009$ in C19, $0.4 \pm 0.01$ in $\mathrm{Ct}, \mathrm{p}<0.001$ ), and a decrease in alpha $(0.12 \pm 0.007$ in $\mathrm{C} 19,0.18 \pm 0.01$ in $\mathrm{Ct}, \mathrm{p}<0.001)$ and beta power $(3.8 \mathrm{E}-02 \pm 0.002$ in $\mathrm{C} 19$, $4.8 \mathrm{E}-02 \pm 0.002$ in $\mathrm{Ct}, \mathrm{p}<0.001)$. Analysis of power in individual 16 channels further showed that significant changes were not localized to particular brain areas, except for a distinct increase in beta in channel $\mathrm{Cz}$.

Furthermore, when power within individual frequency bands was plotted against age, and moving averages of these plots were fit to polynomial trend lines, a crossover in power amplitude was noted around age 70 (Fig 3). Noting that this crossover timeline was approximate (because it was generated based on matching requirements within 5 years of age), age 70 was then used to divide subjects within a group as younger (i.e. below 70 age) or older groups (i.e. above 70 age), and to subsequently test the effect of age on power changes in $\mathrm{C} 19$ patients compared to control.

Results indicate that the power changes observed between age-matched C19 and control groups is further accentuated when the analysis is restricted to only younger subjects (Fig 4), whereby delta and theta are significantly increased in the $\mathrm{C} 19$ group compared to control $(0.5 \pm 0.009$ in $\mathrm{C} 19,0.3 \pm 0.01$ in $\mathrm{Ct}, \mathrm{p}<0.001$ for delta, and $0.24 \pm 0.007$ in $\mathrm{C} 19,0.01 \pm 0.003$ in $\mathrm{Ct}, \mathrm{p}<0.001$ for theta), whereas alpha and beta are significantly decreased $(0.11 \pm 0.006$ in $\mathrm{C} 19,0.21 \pm 0.015$ in $\mathrm{Ct}, \mathrm{p}<0.001$ for alpha, and $0.04 \pm 0.0013$ in $\mathrm{C} 19,0.06 \pm 0.002$ in $\mathrm{Ct}$, $\mathrm{p}<0.001$ for beta). Analysis of power within individual channels did not show localized changes (Sup Fig 2), whereas phase-amplitude coupling between gamma and lower frequency bands was not changed (Sup Fig 3). On the other hand, older $\mathrm{C} 19$ subjects showed a different pattern of power changes relative to younger subjects, such as decreased theta $(0.24 \pm 0.01$ in $\mathrm{C} 19,0.28 \pm 0.008 \mathrm{in} \mathrm{Ct}, \mathrm{p}<0.001)$ as well as increased beta and gamma $(0.036 \pm 0.0012$ in $\mathrm{C} 19,0.028 \pm 0.0014 \mathrm{in} \mathrm{Ct}, \mathrm{p}<0.001$ for beta, and $0.028 \pm 0.001$ in $\mathrm{C} 19$,

$0.018 \pm 0.0007$ in $\mathrm{Ct}, \mathrm{p}<0.001$ for gamma). However, no statistically significant power change was observed at the single channel level (Sup Fig 4). These data prompted a comparison within C19 group between younger versus older subjects, which showed no significant change in power except a decrease in gamma in younger subjects $(0.019 \pm 0.0006$ in younger, $0.024 \pm 0.001$ in older, $p=Z)$, results that were consistent with the lack of statistically significant power change at the single channel level (Sup Fig 5). A summary of mean power changes in all channels is provided in Table 3.

Lastly, machine learning algorithms for the binary classification of subjects as C19 versus control based on EEG recording reached consistently higher accuracy levels when trained on datasets in the younger age population (Table 4), with a mean of $70.5 \%$ accuracy in younger subjects compared to a mean of $55.6 \%$ for older subjects. These values were obtained using EEG power features selected based on the results of statistical significance obtained in these groups (for example mean delta, theta, alpha and beta for younger subjects, and theta, beta and gamma for older subjects). Moreover, when the same feature selection used to train a given algorithm in one group was applied to the other group for direct comparisons of accuracy, algorithms for the younger subjects still performed better than those in older ones $(77.2 \%$ versus 47.5 , and $69.4 \%$ versus $55.6 \%$, respectively).

\section{Discussion}

The neurological symptoms of SARS-CoV-2 range from mild headache to altered mental status and seizures [4-7]. Assessment of these symptoms is based primarily on subjective criteria, self-reported questionnaires, and EEG when clinically indicated. However, the scientific literature on EEG and C19 is almost exclusively based on visual interpretation of EEG waveforms. This is a major concern because artifacts are ubiquitous in EEG and contribute to inter-rater variability and sampling bias [20-22]. Moreover, small sample sizes, EEG analysis only in the time domain, lack of adequate control for the clinical environment with a negative PCR test for $\mathrm{C} 19$, and disregard to age as a significant variable, are all factors that compromise rigor and confound the interpretation of prior studies [14]. Therefore, we implemented an automated analytical pipeline for the analysis of EEG in the frequency domain to investigate neural oscillations in the brains of patients with C19, and compared those to control subjects in the same clinical environment but with a negative C19 diagnosis.

Our visual, qualitative analysis of EEG suggests that diffuse encephalopathy was highly prevalent in C19 patients, as well as in control patients. However, diagnosis was inconsistent between two independent sites at the individual patient level. Although this finding confirms prior reports about the high incidence of $\mathrm{C} 19$ related encephalopathy [8-19], it highlights the challenges of EEG assessment based on visual inspection. We 
presume that the clinical diagnosis of encephalopathy in C19 patients is likely a sequel to C19 for patients admitted to the ICU, rather than a direct cause of the viral infection.

Our objective, quantitative analysis of the EEG data showed that the average power spectrum across EEG channels in C19 patients is significantly enhanced in the delta band, and attenuated in the alpha and beta bands compared to aged-matched control patients in the ICU with a negative C19 PCR test. Corroborating the diffuse characteristics of the encephalopathy, changes within these distinct frequency bands appear to be nonlocalized to individual channels (except in CZ for beta). Moreover, we report that phase-amplitude coupling within individual channels is not significantly changed in C19 patients compared to control, indicating that the EEG changes in power do not necessarily translate to a generalized disruption in cross-frequency coupling between fast (gamma) and slow (delta-to-beta) bands, a proxy for effective brain communication.

These results related to oscillations, obtained in a fully-automated approach that enhances rigor and reproducibility, corroborate prior observations using visual inspection of the EEG, although past studies did not provide consistent and numerical estimates for these changes, nor accurate localization to specific channels and brain areas. According to a systematic review and meta-analysis, the proportion of abnormal EEG in C19 subjects is as high as $96 \%$ [16]. Non-specific, generalized background or diffuse slowing has been reported in several studies as the most common finding $[10,11,18]$, whereby EEG abnormalities correlate with encephalopathy, disease severity [8,9,17], and risk for novel seizures [13], including psychogenic nonepileptic seizures [5]. In particular, delta waves with absence of epileptic activity have been documented [12], as well as alpha coma patterns suggestive of SARS-CoV-2 neurotropism for the brainstem ascending reticular system [15]. Although some studies suggested that SARS-CoV-2 could preferentially and directly target the frontal lobes [19], with EEG abnormalities localized to frontal electrodes [8, 12], our results did not confirm these qualitative observations. The mechanisms underlying these observations are largely unknown; however, it has been suggested that metabolic hypoxia [18], immune-mediated neuro-inflammation [3], as well as severe stress and sleep disturbances [5], are likely contributing factors.

Further analysis revealed evidence for age-dependent variations in power, with a clear threshold for differential effects around 70 years. Analysis of power in the younger age group (below age 70) showed significantly enhanced power in the delta and theta bands, and attenuated power in the alpha and beta bands, compared to the older population (above age 70). These power changes in younger individuals were not localized to individual channels, but more prominent in magnitude when compared to all ages combined. In older subjects, the power patterns were different from those in younger subjects, with significantly attenuated power in theta, and enhanced power in beta and gamma. However, these changes within the older population were less severe compared to those in the younger population, and were not localized to individual channels. Lastly, when power distribution in younger age C19 patients was compared to older ones with C19, no change in power was noted in lower frequency bands below $35 \mathrm{~Hz}$, although gamma was significantly higher in older patients (results summarized in Table 3). To date, no study has investigated the age-dependency of C19 on dynamic brain physiology. Our results highlight the fact that the changes in EEG patterns affect younger subjects more than older ones, which is surprising given the general dogma that older individuals are at higher risk of developing severe C19 symptoms. Vulnerability of pediatric patients with multi-system inflammatory syndrome has been previously documented [26], whereby long-term EEG monitoring after healing is recommended [27]. Hence, our results emphasize the need for continuous, long-term EEG monitoring for C19 patients in the ICU regardless of age, whereby we have shown that EEG power distribution in the low frequency range of patients below age 70 is indistinguishable from that above age 70 . They further raise critical questions about reversibility and the potential long-term effects of C19 in younger individuals.

The results of our data driven and statistically guided machine learning algorithms further demonstrate that the accuracy of binary classification is consistently higher for younger individuals. Machine learning is based on the recognition of patterns and their representation (in our case without prior knowledge of data attributes other than binary labeling attached to C19 positive versus C19 negative input categories). In general, the more clearly delineated patterns are in the features used to train an algorithm (for example separation in numerical values between labels), the higher the algorithm's performance. This suggests that the EEG features in younger adults are more distinct, and changes are more pronounced in C19 positive versus C19 negative labels, whereas in older individuals these features seem to be less distinct between labels. This assumption is well supported by the statistical analysis of the quantitative EEG.

The limitations in this study include the retrospective design, which was constrained by the need to collect EEG data from critically ill patients with a highly infectious disease during a pandemic, and the inability to control important variables such as medication and underlying medical conditions, although it is noteworthy that the significant EEG slowing in this study withstood this variability. Moreover, our conclusions might not generalize to $\mathrm{C} 19$ patients outside of an ICU setting with less severe neurological symptoms. These 
attenuating gaps will be addressed in future prospective studies, whereby our quantitate methodology suggests a valid approach for developing diagnostic, prognostic and therapy monitoring of CNS symptoms secondary to C19 using machine learning and quantitative EEG. In addition, our analytical pipeline may prove useful for the objective and rapid evaluation of EEG findings in individuals who sustain lasting cognitive changes as seen in "long Covid". 


\section{Methods}

Study population

After institutional review board (IRB) approval, we cross-matched the Cleveland Clinic C19 registry with the Cleveland Clinic EEG database (Ebase, Cleveland, OH) from April 20th, 2020 until May 20th, 2020. All hospitalized C19 adults ( $\geq 18$ years of age at the time of diagnosis of C19) who underwent an EEG were included in the study population, excluding patients in coma, stupor or intubation. Patients were excluded if they did not undergo EEG evaluations during their admission for C19 infection. We identified $n=69$ ICU patients ( $n=31 \mathrm{C19} ; \mathrm{n}=38$ Control, age-matched to $C 19$ within 5 years of age) who underwent a 20-minute screening EEG and at least $24 \mathrm{hr}$ continuous EEG monitoring. All C19 patients had at least one SARS-CoV-2 positive test prior to the initiation of EEG or during EEG monitoring. A complete list of underlying conditions and comorbidity for every patient is shown in Table 1.

\section{EEG preprocessing}

Pre-processing of EEG data, feature extraction, statistics, and machine learning were performed using MatLab (MathWorks, Natick, MA). EEGs were collected at a sampling rate of $200 \mathrm{~Hz}$. A high-pass filter with a passband frequency of $1 \mathrm{~Hz}$ and a notch filter with a stop-band of $57.5-62.5 \mathrm{~Hz}$ were applied to all recordings. All EEGs were first visually inspected to confirm signal quality for each channel; channels considered to be of low or irretrievable quality were excluded from the study. Waveforms in each channel were divided into 1second epochs, and each epoch was tested for the presence of artifacts using a previously validated method based on automated detection of artifacts by a SVM [34]. Epochs not containing artifacts were included in further analysis; all other epochs were excluded.

\section{Feature extraction}

From the remaining artifact-free epochs of each recording, the following features were calculated for all channels: band-wise PSD Power Spectral Density (PSD) for all channels, and band-wise Phase-Amplitude Coupling (PAC). To create the band-wise PSD, a periodogram was gathered from artifact-free epochs, and then these periodograms were averaged together for each channel within each subject. These averaged periodograms were normalized by dividing each frequency bin by the sum of all bins from 3 to $30 \mathrm{~Hz}$. The normalized PSD was used to calculate the band-wise PSD by taking the average of all bins within each of the following four frequency bands: Delta (1-4 Hz), Theta (5-9 Hz), Alpha (10-13 Hz), Beta (14-32 Hz), and Low Gamma (33-52 Hz). This yielded 5 PSD features for every channel included. PAC was calculated using the Modulation Index (MI) method [35]. The center frequencies used for phase included all the even numbers from 2 to 20 . The center frequencies used for amplitude included all multiples of 3 from 30 to 54 . MI was measured for each pair of phase and amplitude frequencies (90 total pairs) for each channel, including only those time points for which there were 5 or more consecutive artifact-free eyes-open epochs. This yielded a $9 \times 10 \mathrm{Ml}$ matrix, for every channel of every subject, with each row corresponding to one phase center frequency, and each column corresponding to one amplitude center frequency. This MI matrix was converted to band-wise PAC for the following 3 pairs of bands: Delta-Low gamma, Theta-Low Gamma, Alpha—Low Gamma, and Beta-Low Gamma. Other band-wise PAC were computed for the following 4 pairs of bands: Delta-Medium Gamma, Theta-Medium Gamma, Alpha-Medium Gamma, and Beta-Medium Gamma. This conversion was accomplished by averaging across the appropriate regions of the MI matrix. This yielded 4 PAC features for each channel, and a maximum of 64 PAC features per subject (4 band-pairs x 16 channels).

\section{Statistical analyses}

We used paired two-tailed t-tests to compare the band-wise PSDs between the PCR positive and PCR native age-matched groups [36]. We used two-tailed Wilcoxon rank-sum tests to compare the band-wise PAC from the two groups for each of 4 band pairs and 16 channels (64 tests in total). We chose to use non-parametric statistical test for PAC because values are constrained between 0 and 1, and are therefore less likely to follow a normal distribution, as required by Student's t-test. Statistical significance was established throughout at $p<0.05$. As individual testing was conducted, there was no adjustment for multiple comparisons [37, 38]. Unlike simultaneous (family) testing with a joint null hypothesis comprising two or more null hypotheses, individual testing is utilized to make a decision about one null hypothesis. As each test provides only one opportunity to make a Type I error, the alpha level does not require lowering.

\section{Classification and prediction}

The features described above (band-wise PSD) were used to create a feature-set for training binary classification algorithms. In particular, only mean power values that were shown to be significantly different 
between groups were selected for algorithm training (for example mean delta, theta, alpha and beta for younger subjects, and theta, beta and gamma for older subjects in Fig 1). We validated the classifiers using kfolds cross validation using $k=4$. Classification accuracy was calculated within the k-folds cross validation by counting the number of out-of-sample predicted labels that matched the true label of the sample, and dividing this total by the number of samples ( $n=24$ for age below 70 group and $n=36$ for age above 70 group).

\section{Figure Legends}

Figure 1: (A) Power spectral density (mean of 16 EEG channels) in age-matched control and C19 subjects ( $n=30$ per group) in the $0-50 \mathrm{~Hz}$ frequency range. (B) Mean power in the frequency bands delta (1-4 Hz), theta (5-9 Hz), alpha (10-13 Hz), beta (14-32 Hz) and low gamma (33-52 Hz).

Figure 2: Power spectral density in 16 individual EEG channels in age-matched control and C19 subjects ( $n=30$ per group) in the frequency bands delta $(1-4 \mathrm{~Hz})$, theta $(5-9 \mathrm{~Hz})$, alpha $(10-13 \mathrm{~Hz})$, beta $(14-32 \mathrm{~Hz})$ and low gamma (33-52 Hz). Heat map (bottom right) shows t-test values for individual channels in each band (red hue indicates $\mathrm{p}<0.05)$.

Figure 3: (Left panel) Power values (mean of 16 channels) were plotted as a function of age in control and C19 subjects, superimposed over 3-steps moving averages in each group, for delta, theta, alpha, beta and low gamma bands. Of note, $\mathrm{X}$-axis values represent the average age for each pair of subjects based on the matching requirements of maximum 5 years difference (i.e. X-axis is non-linear). (Right panel) Moving averages shown in left panel were fitted with polynomial trend lines, which were convergent or intersecting around age 70 for alpha, beta and gamma (vertical lines). This formed the basis of group selection by age below and above 70 years.

Figure 4: (A) Power spectral density (mean of 16 EEG channels) in age below 70 (younger) control and age below 70 (younger) $\mathrm{C} 19$ subjects ( $\mathrm{n}=18$ per group). Histograms show power in individual frequency bands. (B) Same as (A) for age above 70 (older) control and age above 70 (older) $C 19$ subjects ( $n=12$ per group). (C) Same as (A-B) for age above 70 (older) C19 subjects $(n=19)$ and age below 70 (younger) C19 subjects $(n=12)$.

Table 1: Summary of clinical comorbidity for each patient (DM: Diabetes Mellitus; HLD: Hypersensitivity Lung Disease).

Table 2: Qualitative assessment of EEG by two independent teams at Cleveland Clinic and SUNY for the diagnosis of encephalopathy $(n=38$ control, $n=31$ C19).

Table 3: Summary of per cent power changes in Figures 1 and 4.

Table 4: (Upper row) Accuracy results of machine learning algorithms trained on EEG power features selected based on statistical tests performed in Figures 1 and 4 (for example in Figure 1 mean delta, theta, alpha and beta for younger subjects, and theta, beta and gamma for older subjects). (Bottom row) Feature sets used to train algorithms in given group were applied to the other group for direct comparison. Only models with accuracy $>50 \%$ are shown.

Supplemental Figure 1: Age distribution for subject pairs with a matching requirement of maximum 5 years difference in $\mathrm{C} 19$ (red) and control groups (black; $\mathrm{n}=30$ subjects per group).

Supplemental Figure 2: Power spectral density in 16 individual EEG channels in age below 70 (younger) control and $\mathrm{C} 19$ subjects ( $n=18$ per group) in the frequency bands delta, theta, alpha, beta and low gamma. Heat map (bottom right) shows t-test values for individual channels in each band (red hue indicates $p<0.05$ ).

Supplemental Figure 3: Phase-amplitude coupling (PAC) in 16 individual EEG channels in age-matched control and $\mathrm{C} 19$ subjects ( $\mathrm{n}=30$ per group) between medium gamma and delta, theta, alpha, beta respectively (upper row), as well as between low gamma and delta, theta, alpha, beta respectively (lower row). No statically significant difference was noted between groups in any individual channel.

Supplemental Figure 4: Power spectral density in 16 individual EEG channels in age above 70 (older) control and $\mathrm{C} 19$ subjects ( $\mathrm{n}=12$ per group) in the frequency bands delta, theta, alpha, beta and low gamma. Heat map 
(bottom right) shows t-test values for individual channels in each band, however, no significant difference was noted.

Supplemental Figure 5: Power spectral density in 16 individual EEG channels in age above 70 (older) C19 subjects $(n=19)$ and age below 70 (younger) C19 subjects $(n=12)$ in the frequency bands delta, theta, alpha, beta and low gamma. Heat map (bottom right) shows t-test values for individual channels in each band, however, no significant difference was noted.

\section{Bibliography}

1. Desforges, M., et al., Neuroinvasive and neurotropic human respiratory coronaviruses: potential neurovirulent agents in humans. Infectious Diseases and Nanomedicine I, 2014: p. 75-96.

2. DosSantos, M.F., et al., Neuromechanisms of SARS-CoV-2: a review. Frontiers in neuroanatomy, 2020. 14: p. 37.

3. Zamani, R., R. Pouremamali, and N. Rezaei, Central neuroinflammation in Covid-19: a systematic review of 182 cases with encephalitis, acute disseminated encephalomyelitis, and necrotizing encephalopathies. Reviews in the Neurosciences, 2021.

$4 . \quad$ Louis, S., et al., Continuous electroencephalography characteristics and acute symptomatic seizures in COVID-19 patients. Clinical Neurophysiology, 2020. 131(11): p. 2651-2656.

5. Rosengard, J.L., et al., Psychogenic nonepileptic seizures during the COVID-19 pandemic in New York City-A distinct response from the epilepsy experience. Epilepsy \& Behavior, 2021. 123: p. 108255.

6. Rastmanesh, R., Sleep EEG Signatures in COVID-19 Survivors: A Facemask/COVID-19-Induced Comment. Sleep and Vigilance, 2021: p. 1-2.

7. Schmidt, C., COVID-19 long haulers. Nature Biotechnology, 2021: p. 1.

8. Antony, A. and Z. Haneef, Systematic review of EEG findings in 617 patients diagnosed with COVID-19. Seizure, 2020.

9. Liotta, E.M., et al., Frequent neurologic manifestations and encephalopathy-associated morbidity in Covid-19 patients. Annals of clinical and translational neurology, 2020. 7(11): p. 2221-2230.

10. Pilato, M.S., et al., EEG Findings in Coronavirus Disease. Journal of Clinical Neurophysiology: Official Publication of the American Electroencephalographic Society, 2020.

11. Roberto, K.T., et al., Electroencephalographic findings in COVID-19 patients: a systematic review. Seizure, 2020.

12. Vespignani, H., et al., Report on electroencephalographic findings in critically ill patients with COVID19. Annals of Neurology, 2020. 88(3): p. 626-630.

13. Gogia, B., et al., EEG Characteristics in COVID-19 Survivors and Non-survivors With Seizures and Encephalopathy. Cureus, 2021. 13(10).

14. Kopańska, M., et al., Changes in EEG Recordings in COVID-19 Patients as a Basis for More Accurate QEEG Diagnostics and EEG Neurofeedback Therapy: A Systematic Review. Journal of Clinical Medicine, 2021. 10(6): p. 1300.

15. Koutroumanidis, M., et al., Alpha coma EEG pattern in patients with severe COVID-19 related encephalopathy. Clinical Neurophysiology, 2021. 132(1): p. 218-225.

16. Kubota, T., P.K. Gajera, and N. Kuroda, Meta-analysis of EEG findings in patients with COVID-19. Epilepsy \& Behavior, 2021. 115.

17. Lin, L., et al., Electroencephalographic Abnormalities are Common in COVID-19 and are Associated with Outcomes. Annals of Neurology, 2021. 89(5): p. 872-883.

18. Sáez-Landete, I., et al., Retrospective Analysis of EEG in Patients With COVID-19: EEG Recording in Acute and Follow-up Phases. Clinical EEG and Neuroscience, 2021: p. 15500594211035923.

19. Toniolo, S., et al., Is the Frontal Lobe the Primary Target of SARS-CoV-2? Journal of Alzheimer's Disease, 2021(Preprint): p. 1-7.

20. Lodder, S.S. and M.J. van Putten, Quantification of the adult EEG background pattern. Clinical neurophysiology, 2013. 124(2): p. 228-237.

21. Duez, C.H.V., et al., Large inter-rater variability on EEG-reactivity is improved by a novel quantitative method. Clinical Neurophysiology, 2018. 129(4): p. 724-730.

22. Ding, J.Z., et al., Resident training and interrater agreements using the ACNS critical care EEG terminology. Seizure, 2019. 66: p. 76-80.

23. Pati, S., E. Toth, and G. Chaitanya, Quantitative EEG markers to prognosticate critically ill patients with COVID-19: a retrospective cohort study. Clinical Neurophysiology, 2020. 131(8): p. 1824. 
24. Pastor, J., L. Vega-Zelaya, and E. Martín Abad, Specific EEG encephalopathy pattern in SARS-CoV-2 patients. Journal of Clinical Medicine, 2020. 9(5): p. 1545.

25. Lee, J.W., COVID-19 EEG studies: The other coronavirus spikes we need to worry about. Epilepsy Currents, 2020. 20(6): p. 353-355.

26. Olivotto, S., et al., Acute encephalitis in pediatric multisystem inflammatory syndrome associated with COVID-19. European Journal of Paediatric Neurology, 2021. 34: p. 84-90.

27. Principi, N. and S. Esposito, Are we sure that the neurological impact of COVID 19 in childhood has not been underestimated? Italian Journal of Pediatrics, 2021. 47(1): p. 1-4.

28. Hughes, J.R. and J.J. Cayaffa, The EEG in patients at different ages without organic cerebral disease. Electroencephalography and clinical neurophysiology, 1977. 42(6): p. 776-784.

29. Nguyen, P., et al. Age and gender classification using EEG paralinguistic features. in 2013 6th International IEEE/EMBS Conference on Neural Engineering (NER). 2013. IEEE.

30. Buzsáki, G. and A. Draguhn, Neuronal oscillations in cortical networks. science, 2004. 304(5679): p. 1926-1929.

31. Allefeld, C., H. Atmanspacher, and J. Wackermann, Mental states as macrostates emerging from brain electrical dynamics. Chaos: An Interdisciplinary Journal of Nonlinear Science, 2009. 19(1): p. 015102.

32. Atasoy, S., et al., Harmonic brain modes: a unifying framework for linking space and time in brain dynamics. The Neuroscientist, 2018. 24(3): p. 277-293.

33. Broyd, S.J., et al., Default-mode brain dysfunction in mental disorders: a systematic review.

Neuroscience \& biobehavioral reviews, 2009. 33(3): p. 279-296.

34. Levitt, J., et al., Automated detection of electroencephalography artifacts in human, rodent and canine subjects using machine learning. Journal of neuroscience methods, 2018. 307: p. 53-59.

35. Tort, A.B., et al., Measuring phase-amplitude coupling between neuronal oscillations of different frequencies. Journal of neurophysiology, 2010. 104(2): p. 1195-1210.

36. Niven, D.J., et al., Matched case-control studies: a review of reported statistical methodology. Clinical epidemiology, 2012. 4: p. 99.

37. Greenland, S., Analysis goals, error-cost sensitivity, and analysis hacking: Essential considerations in hypothesis testing and multiple comparisons. Paediatric and Perinatal Epidemiology, 2021. 35(1): p. 8-23.

38. Rubin, M., When to adjust alpha during multiple testing: a consideration of disjunction, conjunction, and individual testing. Synthese, 2021: p. 1-32. 


\section{Table 1: Clinical comorbidity}

C19 (n=31)

id Comorbidity

1 Cancer, heart murmur

2 EtOH, cirrhosis, acte kidney injury, varices

2 Rheumatoid arthritis

4 DM, psychiatric diagnosis

5 Cardiac arrest

6 HLD

7 Asthma, DM

8 N/A

9 Dyslipidemia, obesity

Heart failure, atrial fibrillation, monoclonal gammopathy of

10 undetermined significance (MGUS), pericardial effusion

11 COPD, headache, recurrent meningitis

12 HLD

13 Atrial fibrillation, DM

14 Prostate cancer

15 DM, heart failure, deep vein thrombosis, hypothyroid, HLD

16 COPD, congestive heart failure, hemicolectomy

17 HLD, glaucoma, AV disorder

18 Peripheral artery disease, osteomyelitis

19 Liver failure

20 Autism, tuberous sclerosis

Cirrhosis, Crohn's disease, vasculitis, hypothyroidism, DM,

21 obstructive sleep apnea, obesity

22 DM

23 Schizoaffective, EtOH

$24 \mathrm{~N} / \mathrm{A}$

25 N/A

26 N/A

27 Alzheimer, bipolar, schizophrenia, hypothermia, DM, HLD

28 Cirrhosis, DM, Gerd

29 HLD

$30 \mathrm{EtOH}$

31 DM, aortic dissection
Ct $(n=38)$

id Comorbidity

1 Transient ischemic attack, HLD, stenosis, carotid artery occlusion

2 Dysphagia, nephrolithiasis

3 Cardiomyopathy

4 Multiple sclerosis

$5 \mathrm{DM}, \mathrm{HPL}$, gout, ca, urinary tract infection

6 End stage renal disease, DM, subdural hematoma

$7 \mathrm{~N} / \mathrm{A}$

8 DM

9 Cirrhosis

10 Atrial fibrillation, HLD, cancer

11 Carotid stenosis

12 Melanoma, bening prostatic hyperplasia, spinal stenosis, cardiomyopathy

13 Multiple sclerosis, depression, anxiety, hypothyroid

14 Chronic Lymphocytic Leukemia, DM, HPL, atrial fibrillation

15 DM, HPL, hypothyroid, COPD, benign paroxysmal positional vertigo

16 Crohn's, COPD, black lung disease, herpes simplex

17 Headache

18 Migraine, depression, postural orthostatic tachycardia syndrome

19 Parkinson disease

20 DM, hepatitis C

HPL, hypothyroidism, paroxismal atrial fibrillation, childhood apraxia of speech,

21 atrial valve replacement

22 Peripheral vascular disease, heart transplant, HLD, DM, hypothyroidism

23 HLD, COPD, giant cell arteritis

24 Parkinson disease, DM

25 Atrial fibrillation, subdural hematoma

Atrial fibrillation, HLD, GERD, complex migraine, vertigo, left common iliac art

26 aneurysm

27 N/A

28 Dementia, depression, hypothoroidism, nephrolithiasis, congestive heart failure

29 Septic arthritis, DM, COPD, asthma, obesity, HLD

$30 \mathrm{HPL}, \mathrm{DM}$, atrioventricular stenosis, atrial fibrillation

31 Cirrhosis, hepatopulmonary syndrome, liver transplant

32 DM, dementia

33 Cardiac arrest

34 N/A

$35 \mathrm{DM}$, Alzheimer disease

$36 \mathrm{~N} / \mathrm{A}$

$37 \mathrm{~N} / \mathrm{A}$

38 Depression 
Table 2: Encephalopathy diagnosis

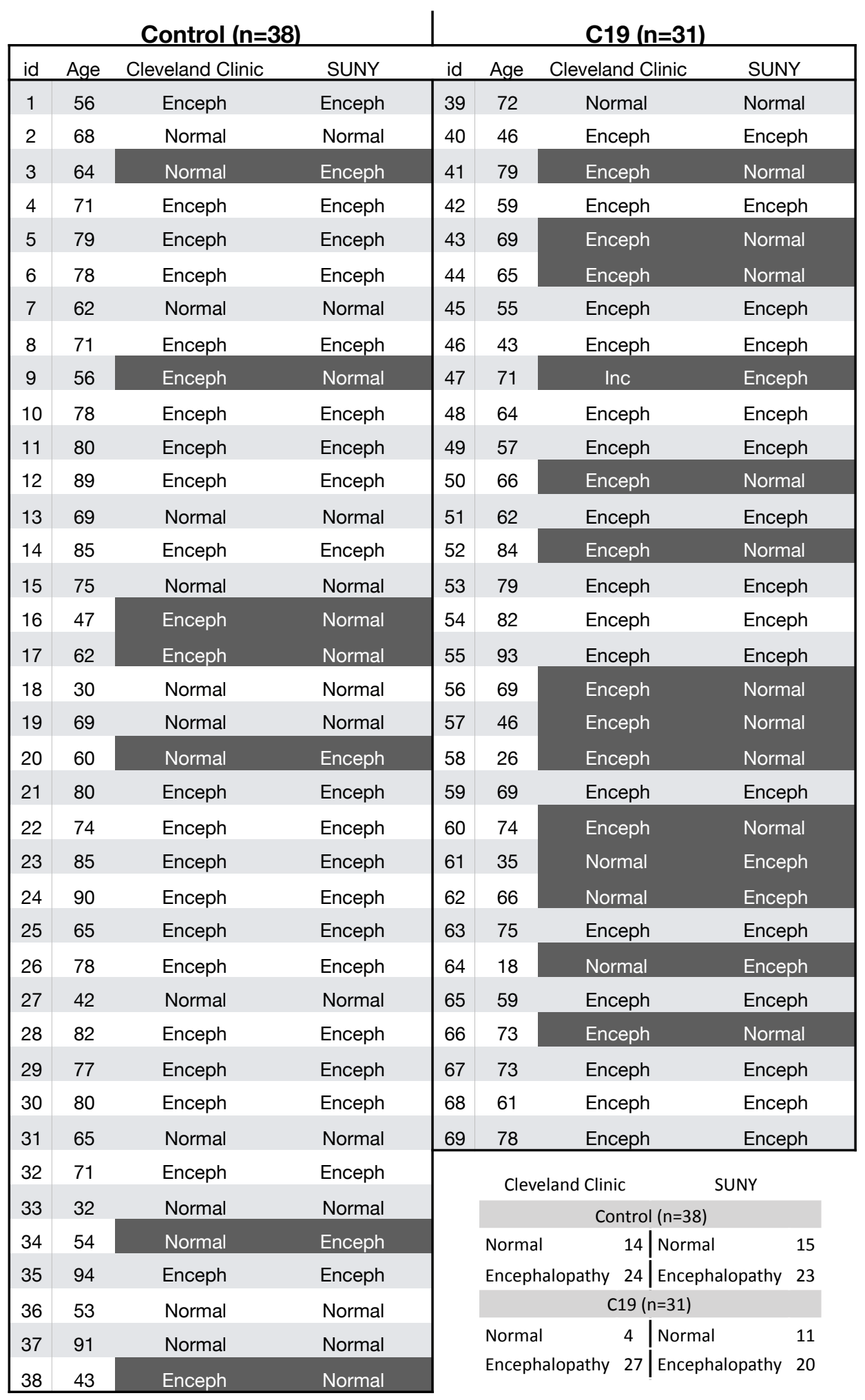


Control versus C19 (all ages, age-matched $\mathrm{n}=30$ )

Power spectra (mean of all channels)

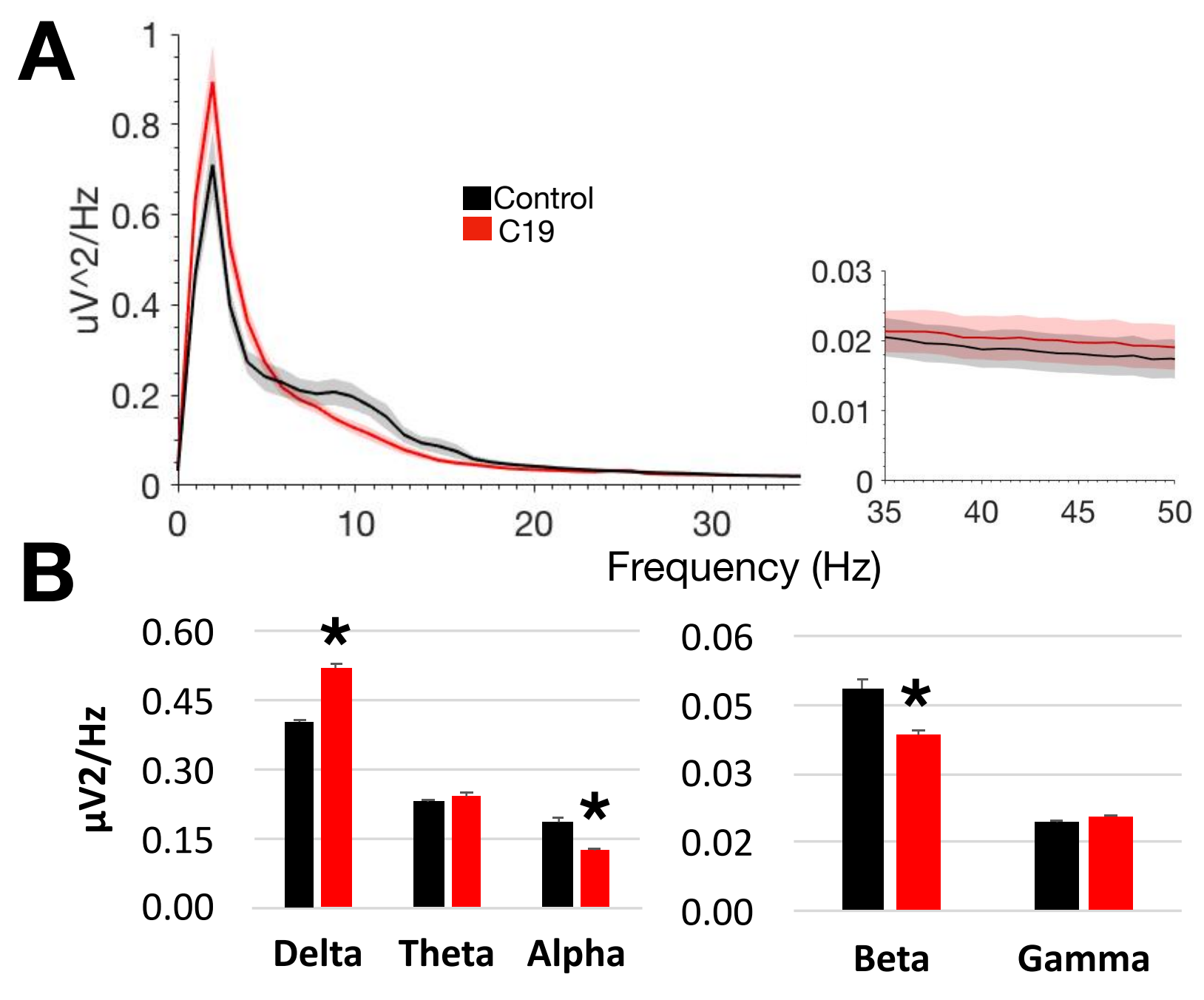

Figure 1 
Control versus $\mathbf{C} 19$ (all ages, age-matched $n=30$ )

Power spectra (individual channels)

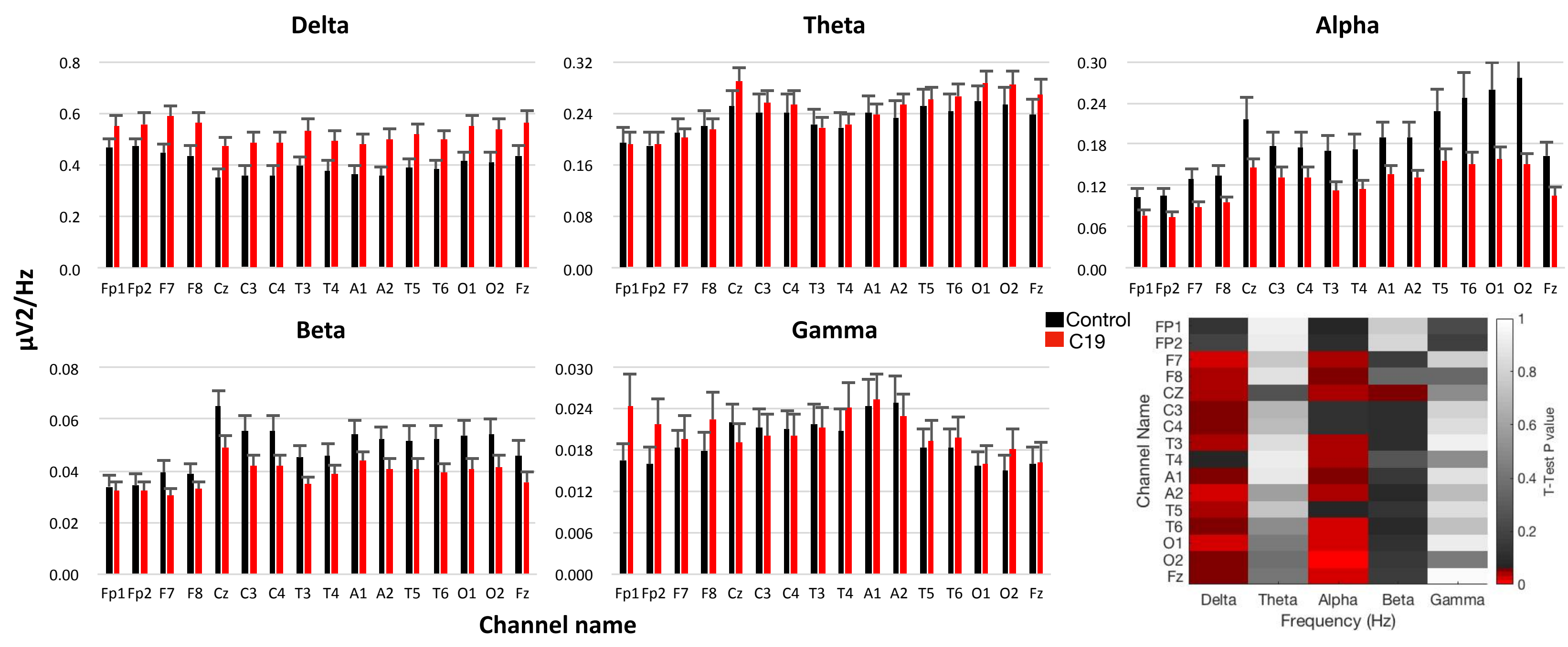

Fig 2 
Power amplitude as a function of age

Control versus C19 (all ages, age-matched $n=30$ )

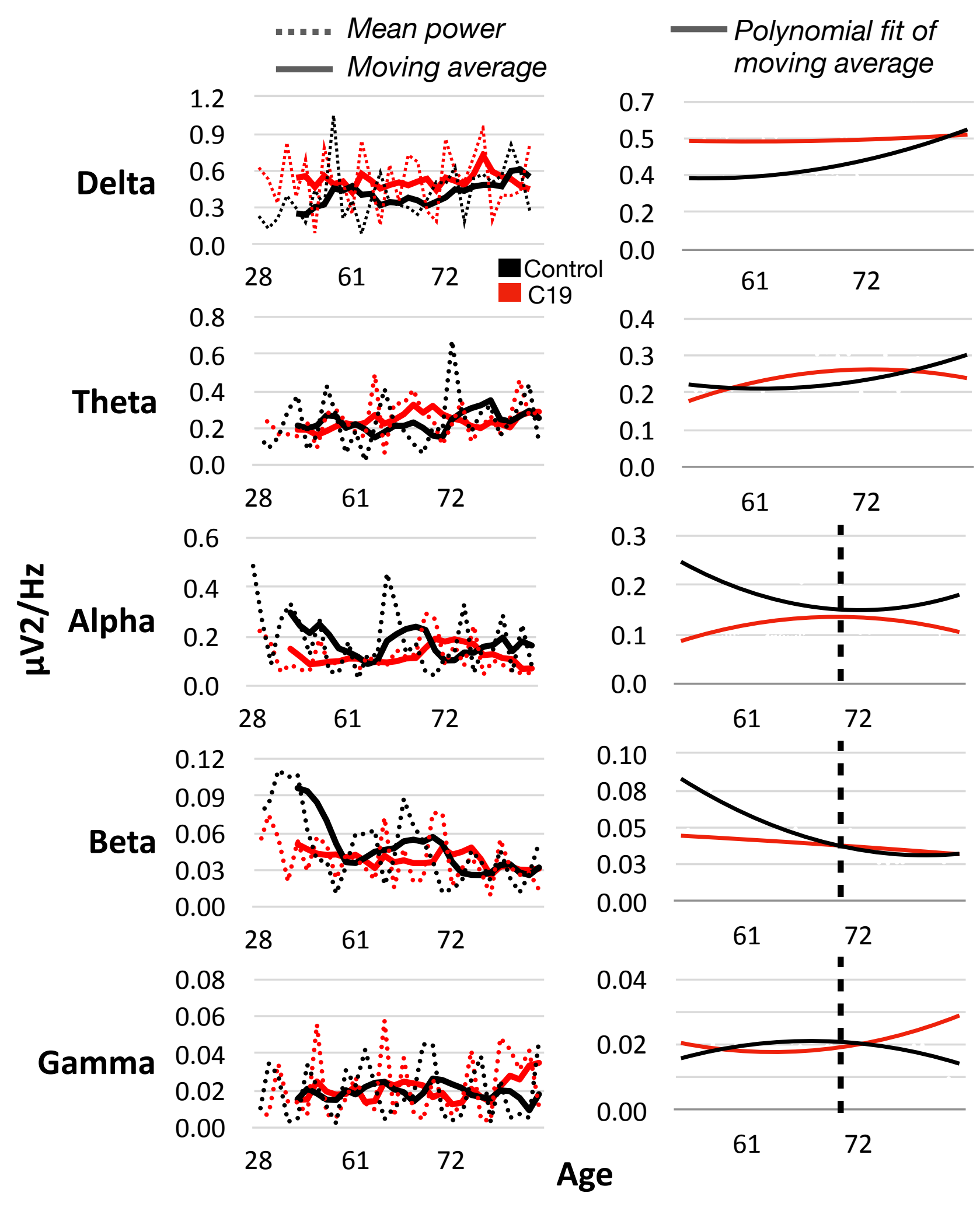

Fig 3 
A

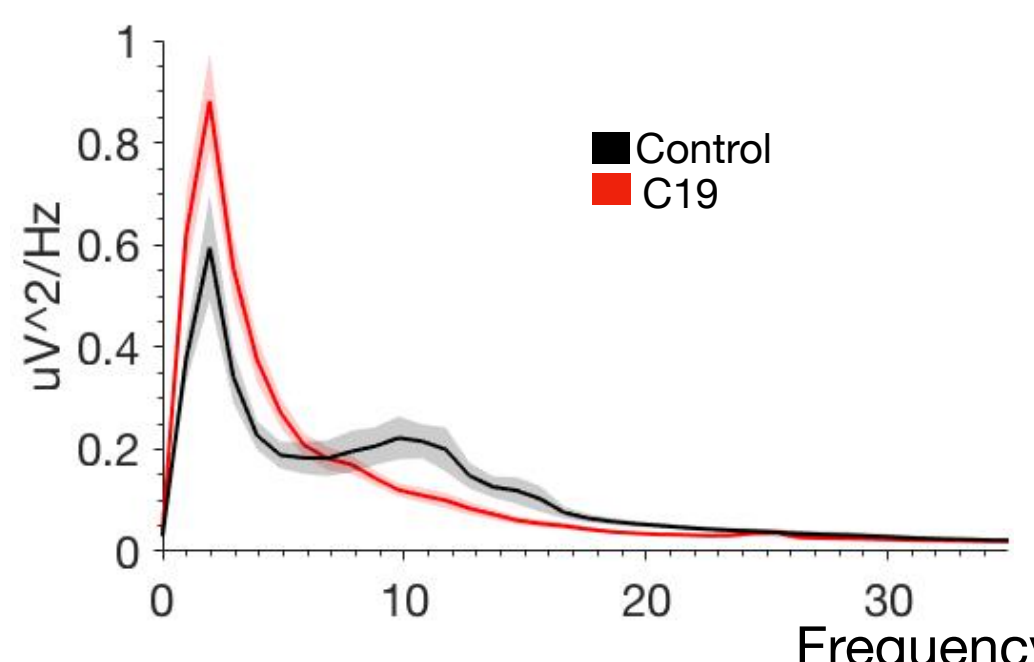

B

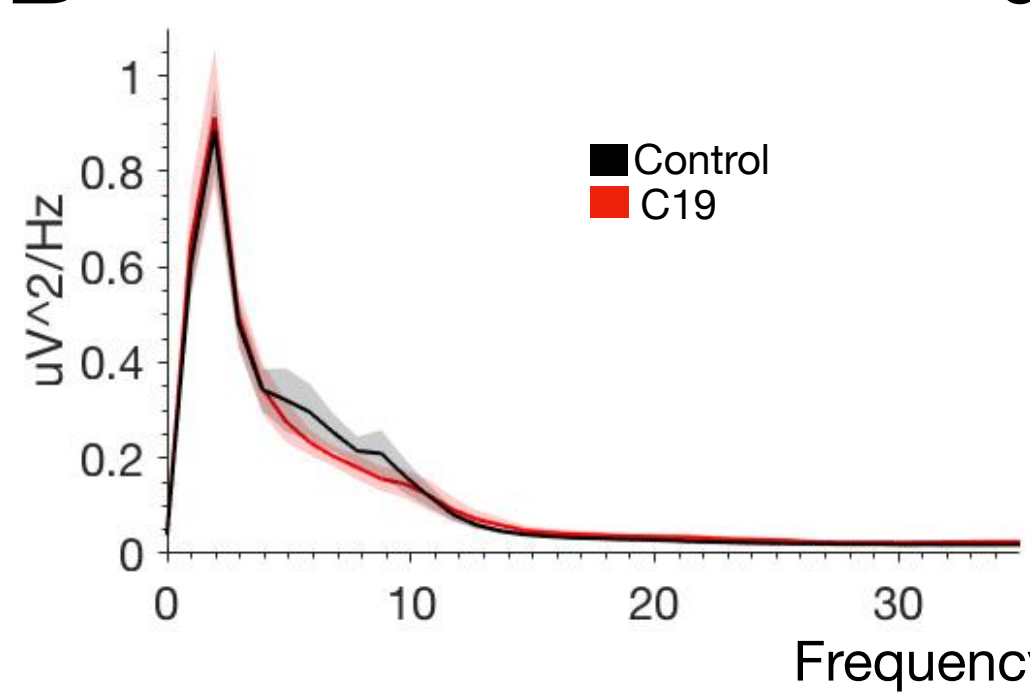

Younger age $C t$ versus younger age $\mathbf{C} 19(n=18)$

Power spectra (mean of all channels)
C

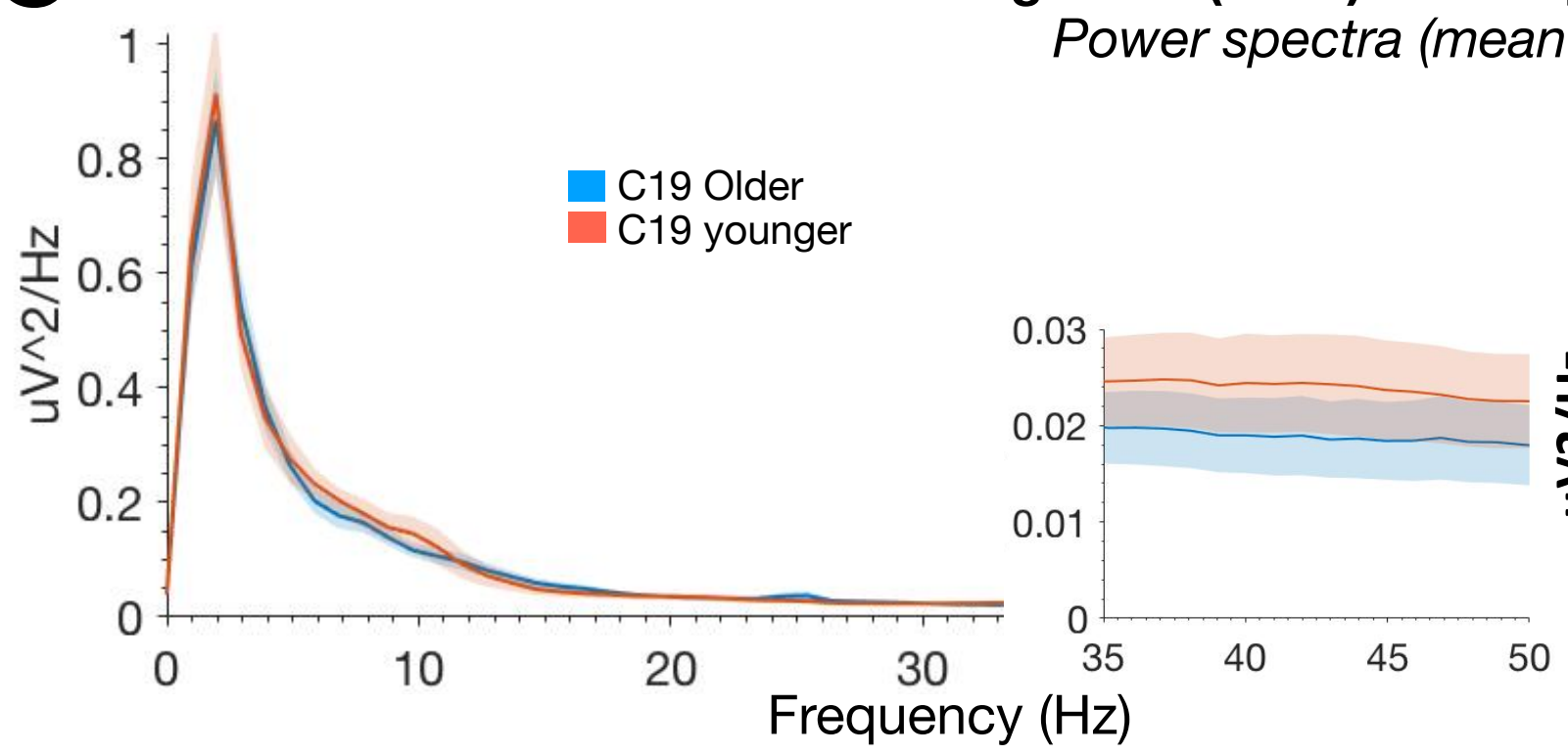

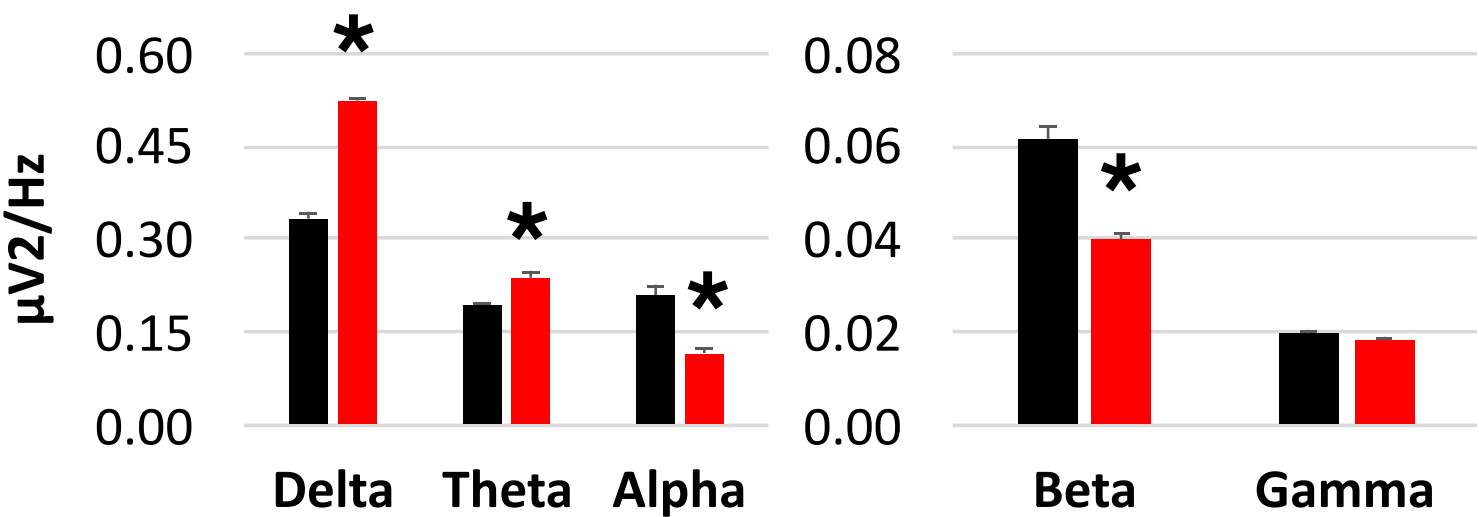

Beta

Gamma

Older age $\mathbf{C t}$ versus older age $\mathbf{C 1 9}(\mathbf{n = 1 2 )}$

Power spectra (mean of all channels)
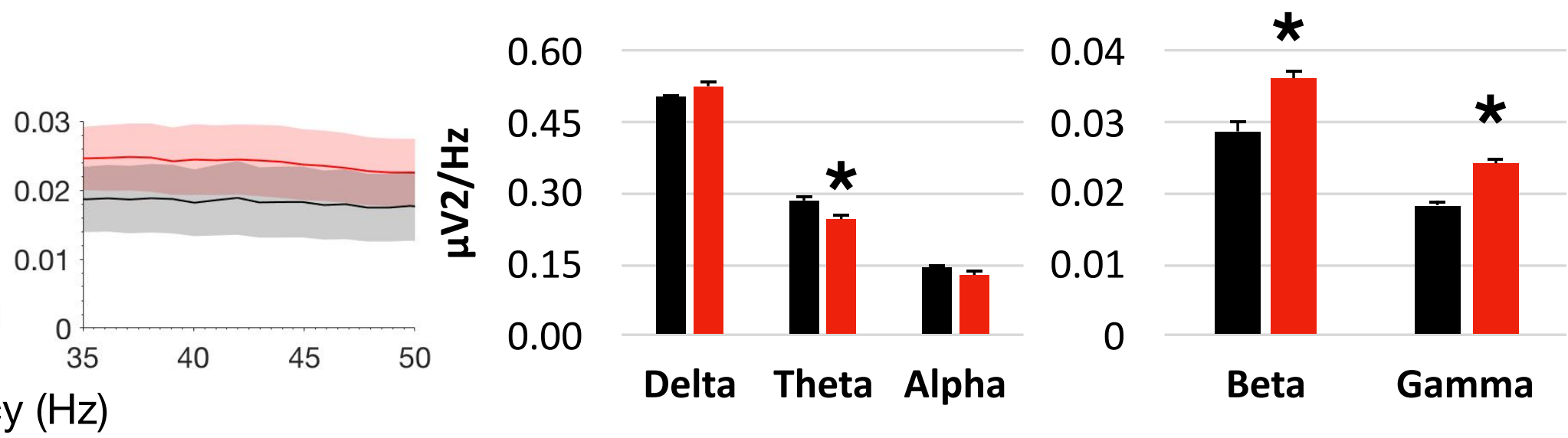

Figure 4

0.06

0.04

0.02

0.00

Delta Theta Alpha

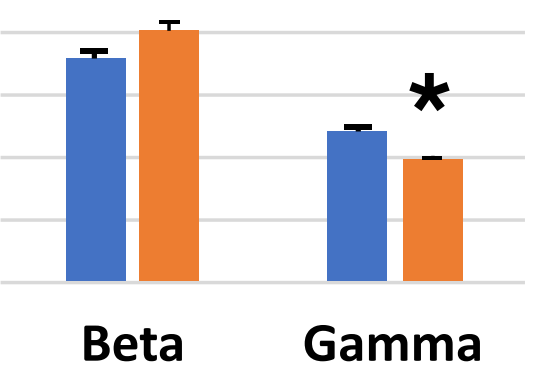




\section{Table 3: Summary of power changes}

\begin{tabular}{|c|c|c|c|c|c|c|c|c|}
\hline \multirow[b]{3}{*}{ Delta } & \multicolumn{2}{|c|}{$\begin{array}{l}\text { Age-matched } \\
\text { Ct vs C19 }\end{array}$} & \multicolumn{2}{|c|}{$\begin{array}{l}\text { Younger } \\
\text { Ct vs C19 }\end{array}$} & \multicolumn{2}{|c|}{$\begin{array}{l}\text { Older } \\
\text { Ct vs C19 }\end{array}$} & \multicolumn{2}{|c|}{$\begin{array}{c}\text { Younger vs older } \\
\text { C19 }\end{array}$} \\
\hline & $\%$ & $+/-$ & $\%$ & $+/-$ & $\%$ & $+/-$ & $\%$ & $+/-$ \\
\hline & 30 & + & 56 & + & ns & 0 & $n s$ & 0 \\
\hline Theta & $n s$ & 0 & 23 & + & -13 & - & $n s$ & 0 \\
\hline Alpha & -33 & - & -43 & - & ns & 0 & $n s$ & 0 \\
\hline Beta & -20 & - & -34 & - & 25 & + & $n s$ & 0 \\
\hline Gamma & ns & 0 & $n s$ & 0 & 30 & + & -19 & - \\
\hline
\end{tabular}




\section{Table 4: Machine learning binary classifiers}

\begin{tabular}{|c|c|c|c|c|c|c|c|c|}
\hline \multicolumn{5}{|c|}{ Younger age $(n=36)$} & \multicolumn{4}{|c|}{ Older age $(n=24)$} \\
\hline \multirow[t]{2}{*}{ Model } & \multirow{2}{*}{$\begin{array}{l}\text { Accuracy } \\
\text { (\%) }\end{array}$} & \multicolumn{3}{|c|}{$\begin{array}{l}\text { Confusion matrix } \\
\text { of top model }\end{array}$} & Model & Accuracy (\%) & \multicolumn{2}{|c|}{$\begin{array}{l}\text { Confusion matrix } \\
\text { of top model }\end{array}$} \\
\hline & & \& $\mathrm{Ct}$ & C19 & & Cubic SVM & 58.3 & $\mathrm{Ct}$ & C19 \\
\hline Coarse Gaussian SVM & 75.0 & $\tau$ & & & Kernel Naïve Bayes & 54.2 & & \\
\hline Gaussian Naïve Bayes & 72.2 & তे 13 & 5 & $\mathrm{Ct}$ & Medium KNN & 54.2 & 8 & 4 \\
\hline Medium KNN & 69.4 & $\frac{0}{0}$ & & & Mean & 55.6 & & C \\
\hline Cubic KNN & 69.4 & 4 & 14 & C19 & & & 6 & 6 \\
\hline Fine Tree & 66.7 & Q True & & & & & Q Trut & \\
\hline
\end{tabular}

\section{Younger age models applied to older age}

\begin{tabular}{|c|c|c|}
\hline Model & $\begin{array}{l}\text { Younger age } \\
\text { accuracy (\%) }\end{array}$ & $\begin{array}{l}\text { Older age } \\
\text { accuracy (\%) }\end{array}$ \\
\hline Coarse Gaussian SVM & 75.0 & 41.7 \\
\hline Kernel Naïve Bayes & 75.0 & 54.2 \\
\hline Gaussian Naïve Bayes & 72.2 & 37.5 \\
\hline Medium KNN & 69.4 & 54.2 \\
\hline Cubic KNN & 69.4 & 50.0 \\
\hline Mean & 72.2 & 47.5 \\
\hline
\end{tabular}

Older age models applied to younger age

\begin{tabular}{l|l|l} 
Model & $\begin{array}{l}\text { Younger age } \\
\text { accuracy (\%) }\end{array}$ & $\begin{array}{l}\text { Older age } \\
\text { accuracy (\%) }\end{array}$ \\
\hline Cubic SVM & 63.9 & 58.3 \\
Kernel Naïve Bayes & 75.0 & 54.2 \\
Medium KNN & \multicolumn{1}{c|}{$\begin{array}{c}\text { M9.4 } \\
\text { Mean }\end{array}$} & \multicolumn{2}{|c|}{$\mathbf{6 9 . 4}$} & $\mathbf{5 5 . 6}$ \\
\cline { 2 - 3 }
\end{tabular}




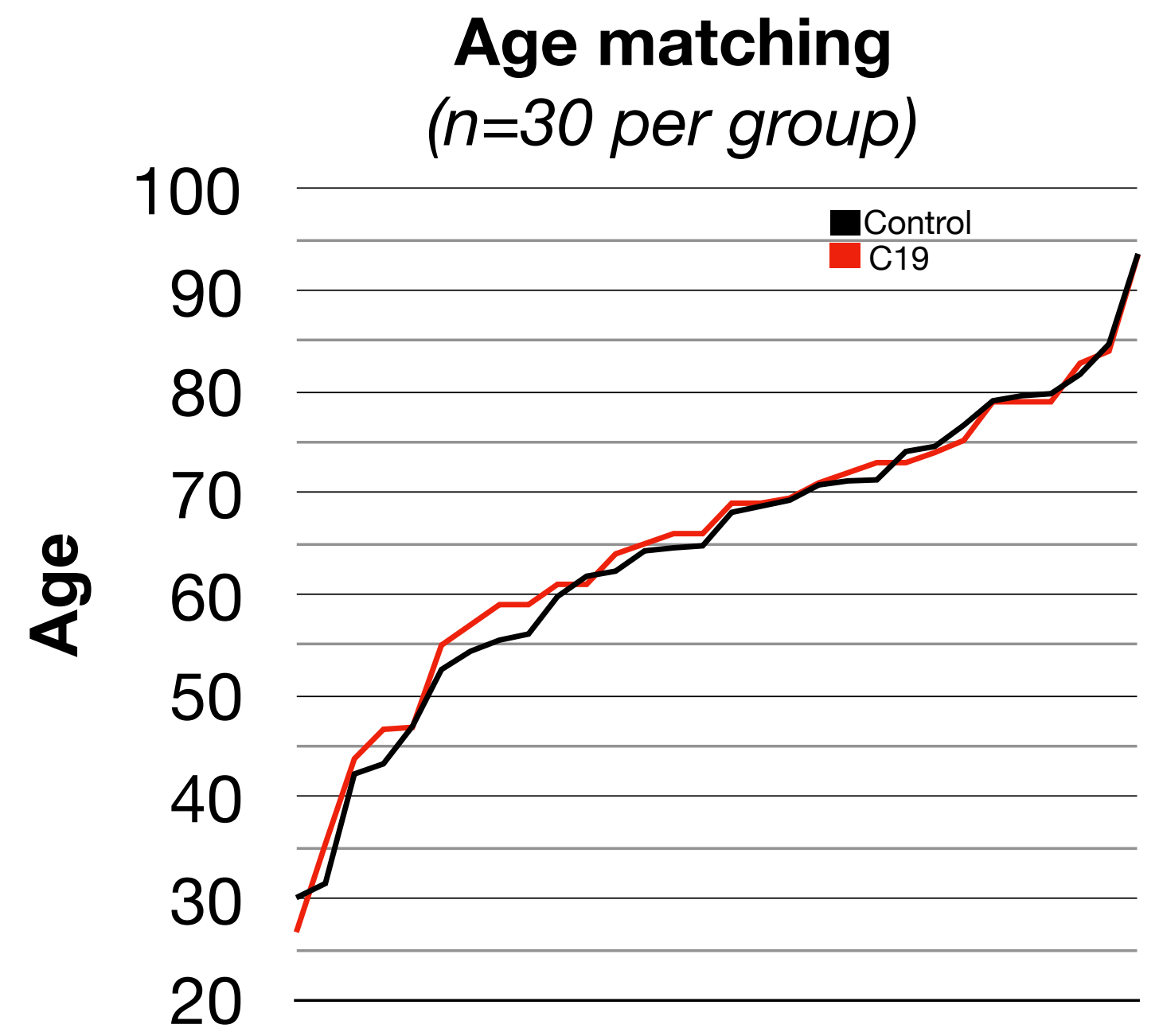

Sup 1 


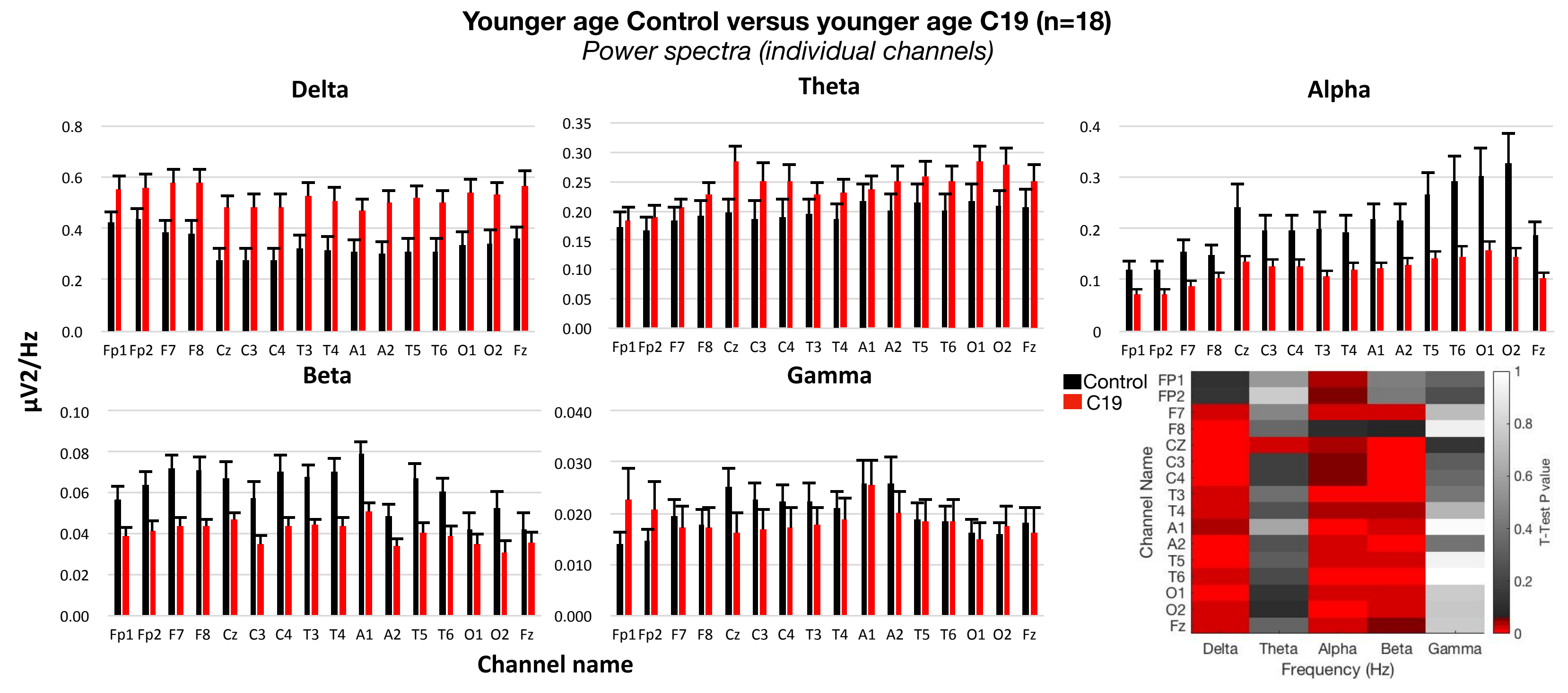


Control versus $\mathbf{C} 19$ (all ages, age-matched $\mathbf{n = 3 0}$ )

Phase-amplitude coupling (PAC)

PAC medium gamma phase with delta amplitude

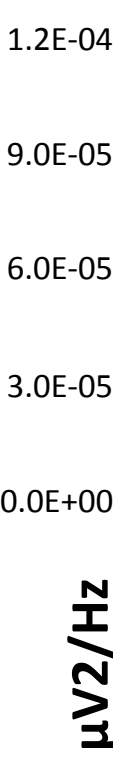

$9.0 \mathrm{E}-05$
$6.0 \mathrm{E}-05$
$3.0 \mathrm{E}-05$
$0.0 \mathrm{E}+00$
ב

$2.0 \mathrm{E}-04$

$1.6 \mathrm{E}-04$

$1.2 \mathrm{E}-04$

$8.0 \mathrm{E}-05$

$4.0 \mathrm{E}-05$

$0.0 E+00$

$$
\text { delta amplitude }
$$

6.0E-05

PAC medium gamma phase with theta amplitude

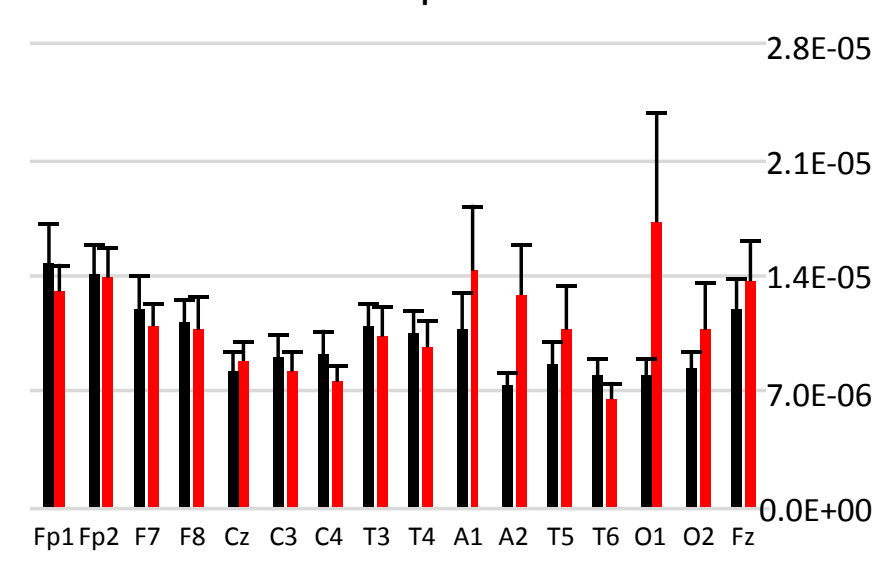

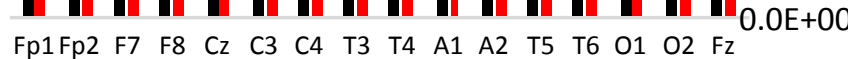
theta amplitude

8.E-05

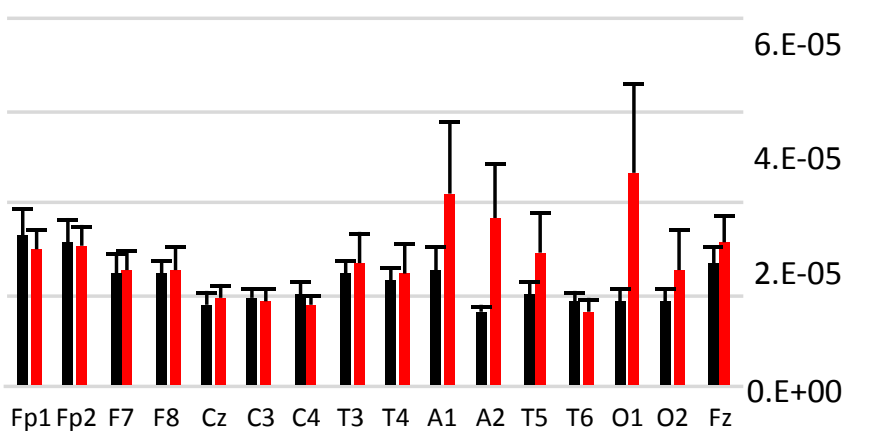

4.0E-05

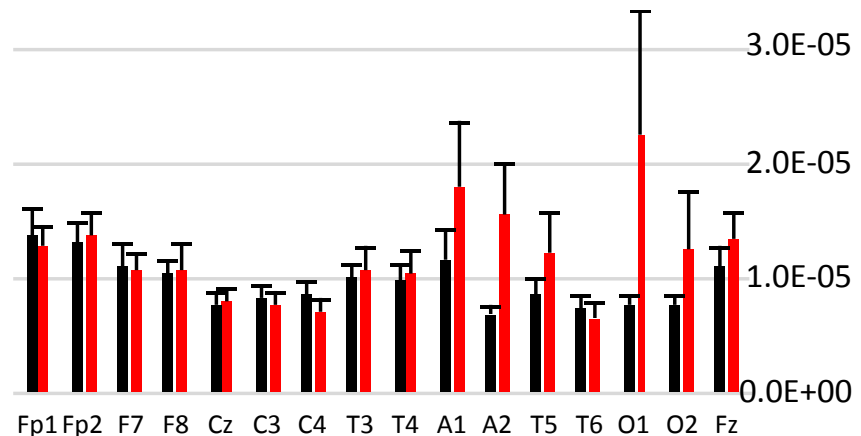

PAC low gamma phase with

PAC medium gamma phase with alpha amplitude

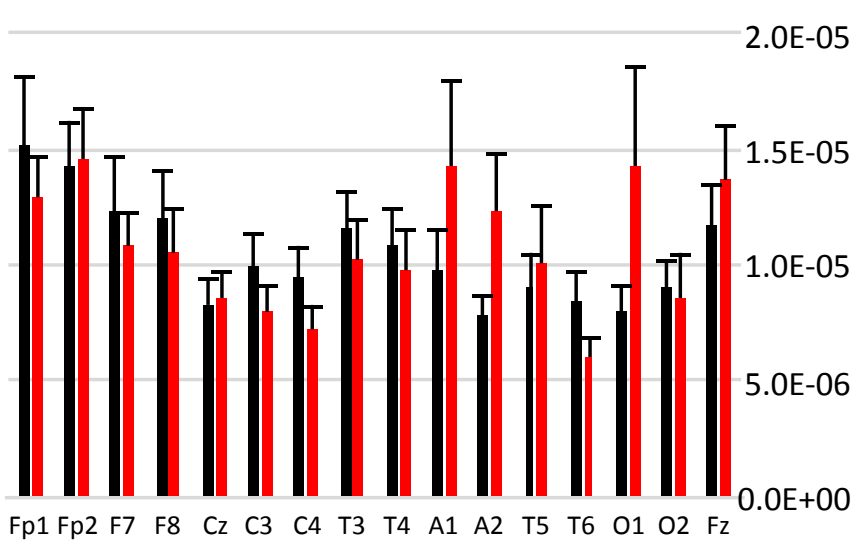

PAC low gamma phase with alpha amplitude

Channel name
PAC medium gamma phase with beta amplitude

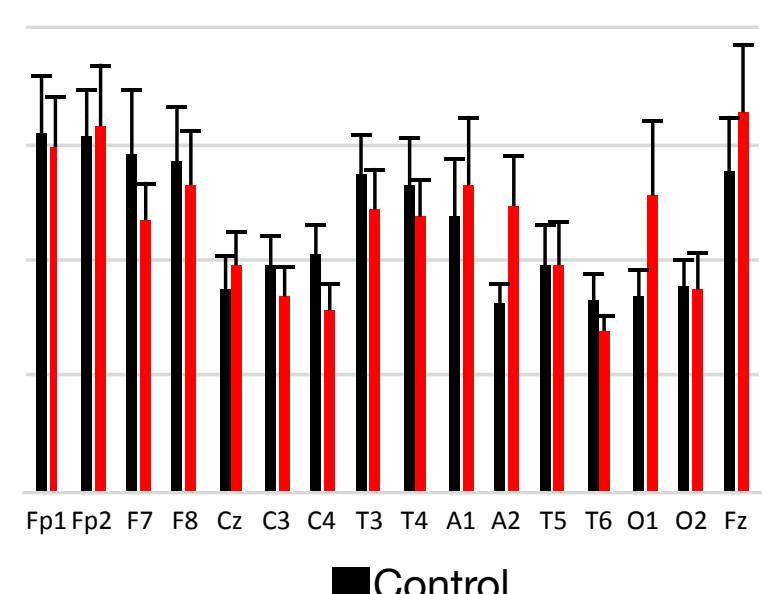

Contro

PAC low gamma phase with beta amplitude

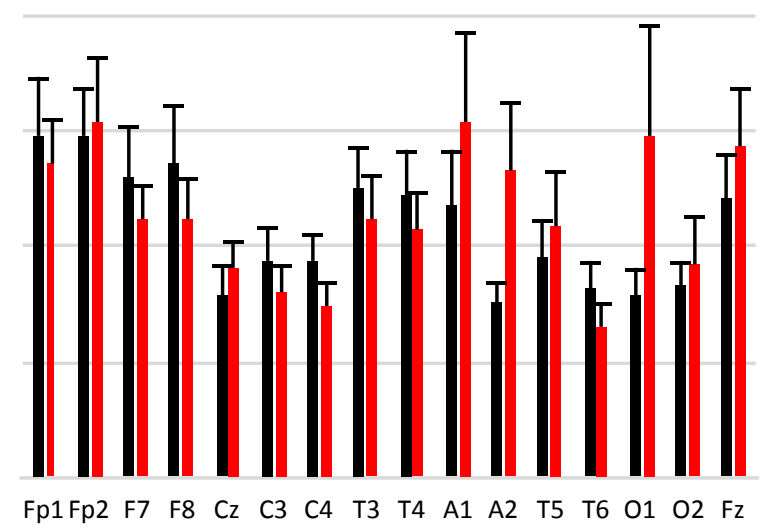

\section{Sup 3}


Older age Ct versus older age $\mathbf{C} 19(\mathbf{n}=12)$

Power spectra (individual channels)

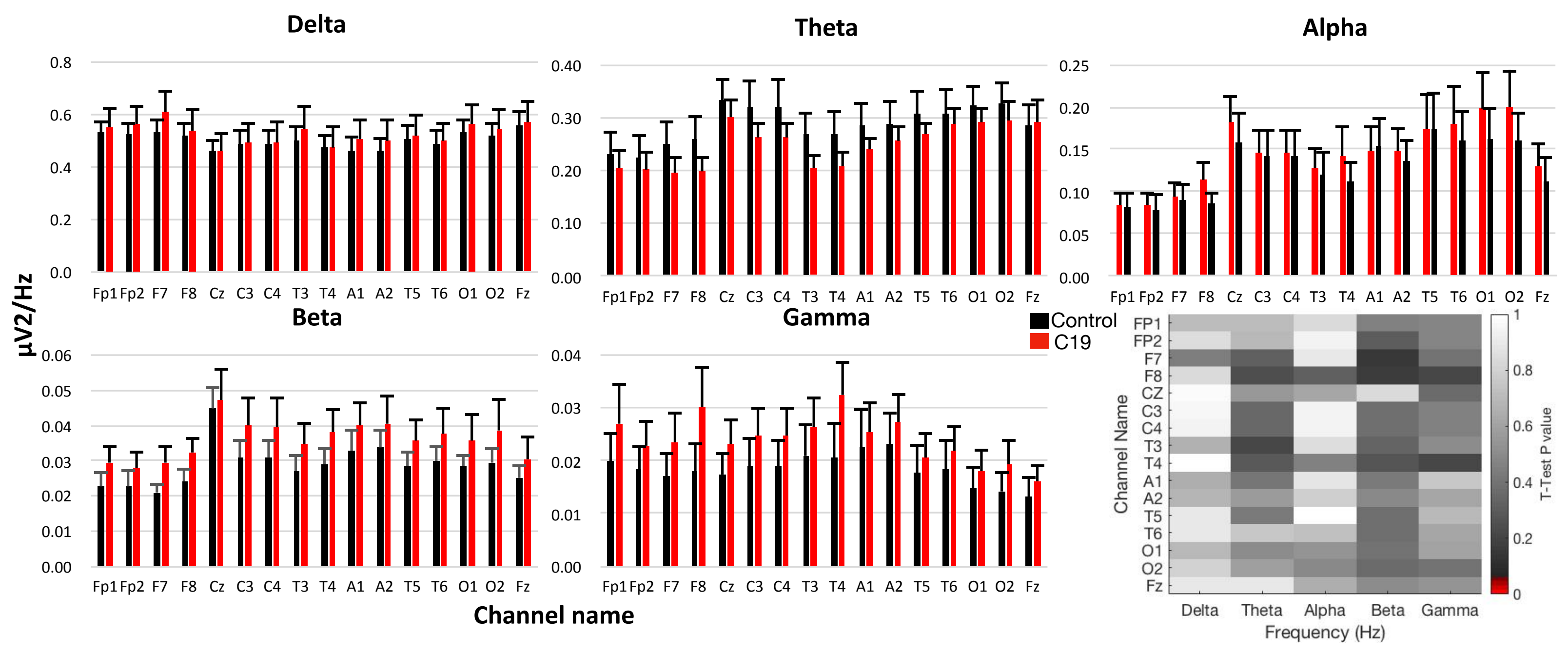




\section{Older age $C 19(n=19)$ versus younger age $\mathbf{C} 19(n=12)$}

Power spectra (individual channels)

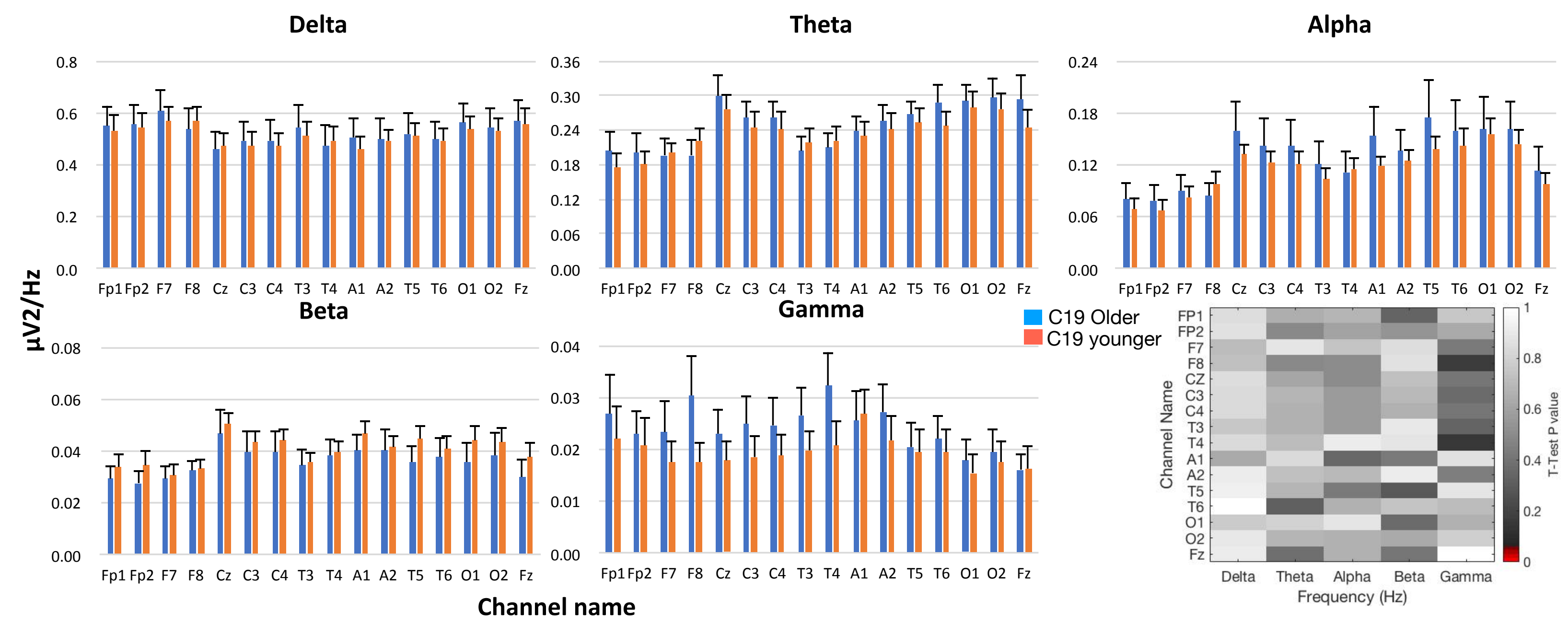

\section{Sup 5}

